\title{
The effect of C-peptide on diabetic nephropathy: a review of molecular mechanisms
}

Habib Yaribeygi ${ }^{1 *}$, Mina Maleki ${ }^{2}$, Thozhukat Sathyapalani ${ }^{3}$, Amirhossein Sahebkar ${ }^{4,5,6 *}$

\author{
${ }^{1}$ Research Center of Physiology, Semnan University of Medical Sciences, Semnan, \\ Iran \\ ${ }^{2}$ Chronic Kidney Disease Research Center, Shahid Beheshti University of Medical \\ Sciences, Tehran, Iran \\ ${ }^{3}$ Department of Academic Diabetes, Endocrinology and Metabolism, Hull York Medical \\ School, University of Hull, Hull HU3 2JZ, UK \\ ${ }^{4}$ Biotechnology Research Center, Pharmaceutical Technology Institute, Mashhad University of \\ Medical Sciences, Mashhad, Iran. \\ ${ }^{5}$ Neurogenic Inflammation Research Center, Mashhad University of Medical Sciences, \\ Mashhad, Iran. \\ ${ }^{6}$ School of Pharmacy, Mashhad University of Medical Sciences, Mashhad, Iran.
}

\section{*Corresponding author:}

Amirhossein Sahebkar, PharmD, Ph.D., Department of Medical Biotechnology, School of Medicine, Mashhad University of Medical Sciences, Mashhad, Iran, P.O. Box: 91779-48564, Iran. Tel: 985118002288; Fax: 985118002287; E-mail: $\quad$ sahebkara@mums.ac.ir; amir_saheb2000@yahoo.com

Habib Yaribeygi, Assistant Professor, Research Center of Physiology, Semnan University of Medical Sciences, Semnan, Iran, Tel: +989355644190; ORCID: 0000-0002-1706-6212

Running Title: C-Peptide and Diabetic Nephropathy

(C2019, Elsevier. This manuscript version is made available under the CC-BY-NC-ND 4.0 license http:// creativecommons.org/licenses/by-nc-nd/4.0/ 


\begin{abstract}
C-peptide is a small peptide connecting two chains of proinsulin molecule and is dissociated before the release of insulin. It is secreted in an equimolar amount to insulin from the pancreatic beta-cells into the circulation. Recent evidence demonstrates that it has other physiologic activities beyond its structural function. C-peptide modulates intracellular signaling pathways in various pathophysiologic states and, could potentially be a new therapeutic target for different disorders including diabetic complications. There is growing evidence that c-peptide has modulatory effects on the molecular mechanisms involved in the development of diabetic nephropathy. Although we have little direct evidence, pharmacological properties of c-peptide suggest that it can provide potent renoprotective effects especially, in a c-peptide deficient milieu as in type 1 diabetes mellitus. In this review, we describe possible molecular mechanisms by which c-peptide may improve renal efficiency in a diabetic milieu.
\end{abstract}

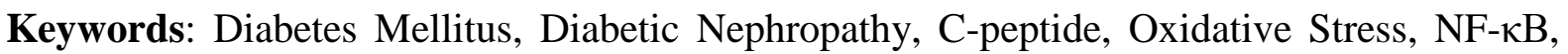
Nitric Oxide. 


\section{Introduction}

The prevalence of diabetes mellitus (DM) is growing exponentially worldwide [1]. It is now the more prevalent metabolic disorder with increasing incidence due to the adoption of a Western and sedentary life-style, especially among younger adults [2, 3]. This chronic disorder has a negative effect on all aspects of metabolism leading to the production of various toxic byproducts [3-6]. DM is responsible for various forms of tissue dysfunction known as diabetic complications $[4,5]$. Diabetic nephropathy (DN) is one of the most important diabetes-induced complications which occur in an uncontrolled diabetic milieu and is considered as the primary cause of the end-stage renal disease (ESRD) [7, 8]. Emerging studies have studied the underlying molecular mechanisms of DN, however, its exact pathophysiology is still unclear $[7,9]$.

The connecting or C-peptide (CP), is a short polypeptide consisting of 31 amino-acids that connects the A chain of proinsulin molecule to the B chain [10]. However, beyond the structural roles in the proinsulin molecules, some evidence suggests that CP can exert metabolic effects and hence may be considered as a potential new therapeutic target for diabetes as well as for various complications of diabetes [10-12]. The CP can bind to various types of cell surface receptors and modulate various intracellular signaling involved in the metabolic pathways and tissue function [10, 13]. Also, some evidence has implied that CP can suppress various molecular mechanisms involved in the pathophysiology of DN and thereby prevent the onset and progression of diabetes-induced renal failure $[10,14,15]$. In the current study, we have reviewed the potential renoprotective effects of CP.

\section{Classification of Diabetes Mellitus}

Type 1 diabetes (T1DM) and type 2 diabetes (T2DM)) are the two common forms of diabetes mellitus (DM) [16]. Type 1 diabetes (T1DM) accounts for about 5-10\% of all patients with diabetes and mainly results from autoimmune destruction of beta-cells of the pancreas [16]. 
Type 2 diabetes (T2DM) is the most prevalent type of diabetes, which accounts for about 9095\% of patients with diabetes and is mainly due to inadequate response to insulin and insulin resistance in the peripheral tissues [16]. Gestational diabetes occurs in pregnant women due to hormonal variations in pregnancy and insulin resistance [17]. The maturity-onset diabetes of young, latent autoimmune diabetes in adults and secondary diabetes due to various medications such as corticosteroids or pathologies such as pancreatitis are some of the other forms of diabetes [16].

\section{Physiological properties of the C-Peptide}

CP was first discovered in 1967, when the insulin biosynthesis pathway was described by Givol [18]. CP has 31 amino-acids with $\mathrm{C}_{112} \mathrm{H}_{179} \mathrm{~N}_{35} \mathrm{O}_{46}$ formula, a molecular weight of 3,020.29 g/mol and a half-life of about 20-30 min and thereby, has more prolonged physiological activity compared to insulin which has a half-life of about 3-5 $\min [19,20]$. CP facilitates the assembly, folding and processing of the insulin peptide in the endoplasmic reticulum organelle. Equimolar amounts of CP and insulin are stored in the secretory granules of the pancreatic beta cells, which are both released in response to various stimuli into the circulation $[18,21]$. Hence the circulating level of CP is closely related to the circulatory level of insulin $[10,11]$.

Recent evidence has demonstrated that the CP is not an inert peptide with only structural functions, but also exerts some biological activities [20]. It has now shown that the CP can bind to some of the cellular membranes such as endothelial cells, pancreatic beta cells, renal tubular cells and fibroblasts; possibly via a specific type of G-protein dependent receptor (a pertussis toxin-sensitive one) and then activates a series of intracellular signaling pathways [20, 22]. This receptor is not sensitive to insulin or insulin-like growth factors (IGF-I and II), suggesting its specificity for CP $[14,23]$. It has also been shown that CP can modulate nuclear events and gene transcription by binding to specific promoters and thereby has an effect on cell survival 
$[20,24]$. Moreover, CP has some degree of insulin-mimetic effects which enables it to modulate glucose metabolism and hyperglycemia as well as diabetes-related complications such as diabetic neuropathy and nephropathy [20-22]. While its plasma concentration is similar to that of insulin level in healthy subjects, CP is absent in T1DM, but exist in T2DM with varying concentrations depending on the stage and progression of DM [14]. The main recognized biological activities of $\mathrm{CP}$ are summarized in table 1.

\begin{tabular}{|c|c|}
\hline Physiologic Functions of C-Peptide & Ref. \\
\hline Structural bridges between A and B chains of proinsulin molecule & {$[20]$} \\
\hline Stimulates $\mathrm{Na}^{+}-\mathrm{K}^{+}$-ATPase expression/activity & {$[25]$} \\
\hline Modulates eNOS and iNOS activities and thereby hemodynamic states & {$[20,26]$} \\
\hline Induces intracellular signaling especially Ca ${ }^{2+}$-dependent pathways & {$[20,26]$} \\
\hline Modulates gene transcription/expression & {$[20]$} \\
\hline Modulates glucose metabolism & {$[20]$} \\
\hline Effects on cell survival & {$[10]$} \\
\hline Modulates thrombotic events & {$[27,28]$} \\
\hline Modulates lipid metabolism & {$[29-31]$} \\
\hline
\end{tabular}

Table 1; known physiological functions of c-peptide (eNOS=endothelial nitric oxide synthase, iNOS=inducible nitric oxide synthase)

\section{Pathophysiology of diabetic nephropathy at a glance}

Diabetic nephropathy (DN), which is the major cause of renal failure worldwide, has complicated and multi-factorial pathophysiology [32-34]. While many clinical studies have assessed its underlying causes, the exact pathophysiology of DN is remaining unclear [35-37]. However, activation of various molecular pathways such as oxidative stress [38], reninangiotensin system (RAS) [39], transforming growth factor- $\beta$ (TGF- $\beta$ ) [40], protein kinase C (PKC) [39], tumor necrosis factor- $\alpha$ (TNF- $\alpha$ ) [39], nuclear factor-kappa-b (NF-кB) [41], adhesion molecules up-regulation [42] and apoptotic events [38, 43] are closely related to different aspects of DN. Other pathophysiological mechanisms, such as hemodynamic 
variations and intraglomerular hypertension, are responsible for a part of tissue injuries in kidneys during the development of DN [44][35, 37].

\section{C-Peptide and renal tissues; possible effects in diabetic nephropathy}

Studies have demonstrated that CP can modulate various intracellular signaling in kidneys [24]. A large number of intracellular mediators and pathways in renal tissues such as PKC (protein kinase c), ERK (extracellular-signal-regulated kinase), JNK (Janus kinase), TGF- $\beta /$ Smad, iNOS, PPAR- $\gamma$ and phosphoinositide 3-kinase are under the influence of the CP activity [4549]. CP can also exert direct regulatory effects on various elements of the nephrons, especially glomerulus and proximal tubule and thereby modulate renal function [14, 45, 50][14]. Evidence suggested that CP may provide renoprotective effects by ameliorating histological damages such as glomerular hypertrophy, mesangial expansion, tubulointerstitial inflammation, tubular sodium reabsorption and glomerular hyperfiltration leading to lowering of albuminuria [5155]. It may also suppress various apoptotic processes and tissue damages in renal tissues in the diabetic milieu $[14,56]$.

Based on these observations, some evidence suggests that CP may be beneficial for the prevention of diabetes-induced pathophysiologic conditions such as DN, especially in patients with T1DM [14, 24, 55, 57]. Evidence demonstrates that patients with T1DM who have some functional pancreatic $\beta$-cells are substantially less susceptible to vascular complications than patients who lose their functional $\beta$-cell and thereby are CP deficient $[58,59]$. In the following sections, we review the possible renoprotective effects of $\mathrm{CP}$ and discuss its therapeutic potential in the diabetic milieu for improvement in renal function and the prevention of DN.

\section{C-peptide and NF-кB}

NF- $\kappa \mathrm{B}$ is an evolutionarily conserved protein from the Rel family which is widely expressed in most cell types and controls the expression of various genes involved in different cellular processes such as the inflammatory responses, cell development, cellular growth and cell 
survival as well as responses to numerous stimuli such as free radicals, stress, cytokines, ultraviolet radiations and invader viral and bacterial antigens [60, 61]. NF- $\mathrm{B}$ hyper-activation is commonly observed in most cases of $\mathrm{DN}$ and is responsible for various forms of renal injuries $[60,61]$. This nuclear element is widely expressed in different segments of the renal tissues, especially in the cortical tubular, epithelial, glomerular and interstitial cells. It can up-regulate various factors involved in the pathophysiology of DN, such as inflammatory mediators, TGF$\beta, \mathrm{Bcl} 2$ and pro-oxidant enzymes and thereby, many studies have considered it as one of the primary therapeutic targets for $\mathrm{DN}[41,60,62,63]$. These studies have demonstrated that the modulation of NF- $\mathrm{BB}$ activity may ameliorate the onset and the progression of various deleterious pathways involved in DN [41, 60, 62].

There is evidence suggesting that CP could potentially modulate NF- $\mathrm{BB}$ activity [64]. CP can regulate NF- $\mathrm{BB}$ activity in different tissues including the kidneys [24, 56, 64]. Cifarelli and coworkers in 2008 found that CP provides protective effects for vascular networks by inhibiting the NF- $\kappa \mathrm{B}$ translocation and activity in the diabetic milieu [64]. Li et al. in 2018 demonstrated that CP could attenuate the progression of DN by inhibition of NF- $\mathrm{BB}$ activity and thereby its down-stream events [24]. Hills and coworkers in 2010 reported that modulation of NF-kB could ameliorate the renal damages in diabetes [56]. Al-Rasheed and colleagues in 2006 demonstrated that the anti-apoptotic effects of $\mathrm{CP}$ are dependent on the NF- $\mathrm{BB}$ modulatory potentials in the renal tissues [65]. They suggested that CP-induced NF- $\kappa \mathrm{B}$ activation protects the renal cells against the TNF- $\alpha$-induced injury in the diabetic milieu [65]. Luppi and coworkers in 2008 have found that the CP therapy ameliorates the diabetes-induced endothelial dysfunction, which commonly occurs in DN [66]. This evidence suggested that CP replacement therapy may be beneficial against vascular disorders in patients with diabetes, especially TIDM [67]. Therefore, we can consider the modulatory effects on NF- $\mathrm{B}$ as a possible molecular mechanism by which CP improves renal efficiency in the diabetic milieu [24, 56, 65-67]. 


\section{Anti-Apoptotic Effects}

Apoptotic and fibrotic events have a pivotal role in the histological damages observed in DN $[38,43]$. It has been shown that these deleterious processes are responsible for the diabetesinduced histopathological injuries in various kidney tissues such as glomerulus and tubular cells and thereby, attenuating their progress is one of the main targets in the prevention and treatment of $\mathrm{DN}[38,68]$. There is strong evidence implying that $\mathrm{CP}$ can suppress these deleterious pathways and reduce the histological injuries in different tissues, especially in T1DM [56, 69, 70]. Li et al. in 2002 reported that $\mathrm{CP}$ reduced the Bax/Bcl-xl ratio and declined apoptosis in the hippocampal tissues of animals with T1DM [70]. Sima and coworkers in 2005 found that CP therapy markedly reduces the apoptotic events and neuronal death in the hippocampal tissues and thereby improves cognitive function in T1DM [71]. Also, Al-Rasheed et al. in 2006 reported that CP attenuates DM-induced apoptosis in kidneys [65]. Cifarelli et al. in 2011 have shown that CP ameliorates the DM-induced apoptosis in endothelial cells by lowering RAC-1 (ras-related C3 botulinum toxin substrate 1) translocation to the membrane and by Nox activation in the human aortic endothelial cells [72]. Bhatt and coworkers in 2013 provided further evidence suggesting that CP protects endothelial cells against DM-induced apoptosis and reduces tissue damages by a transamidation-dependent pathway [73]. In short, although most evidence has demonstrated the anti-apoptotic potentials of CP in the neuronal networks [70, 71], fewer studies indicated that it could exert the same effects in renal tissues and thereby prevent cellular death, especially in T1DM $[65,73]$. However, more experimental studies are still needed to confirm this finding.

\section{Vasoprotective Effects}

DN can be considered as a vascular disorder with dysfunctional vessel walls, hemodynamic variations and lower efficiency of renal vascular networks $[74,75]$. In this state, most renal vessels in glomerulus such as afferent and efferent arterioles are negatively affected by 
hyperglycemia-induced pathophysiologic pathways and thereby loss of their normal physiologic functions [74, 75]. Therefore, some therapeutic targets for DN have been developed based on vasoprotective potentials $[74,76]$.

There is evidence indicating that $\mathrm{CP}$ exerts potent vasoprotective effects in a diabetic milieu [69]. The earliest observation was by Johansson and coworkers in 1992, demonstrating that CP therapy increases the oxygen uptake and blood flow in muscles of patients with T1DM [77]. This evidence suggests that CP improves the vascular function of muscles in diabetic milieu [77]. Hansen and coworkers in 2002 have provided similar evidence in myocardial tissues indicating CP improves blood flow in the myocardium in patients with T1DM [78]. Further studies have confirmed these findings and suggested that CP-induced vasoprotective influences are related to the nitric oxide (NO) synthesis [79-83]. The CP potentially induces eNOS and iNOS activities and thereby increases NO bioavailability leading to an improved vascular tone and vascular efficiency [79, 81-83]. However, other possible pathways are also suggested [84]. Bhatt and coworkers in 2013 reported that CP-dependent vasoprotective potentials are partially related to the AMPK $\alpha$ (AMP-activated protein kinase $\alpha$ ) pathways [84]. They observed that CP replacement therapy declined the AMPK $\alpha$ dephosphorylation in aortic vessels of diabetic animals [84]. Also, as mentioned earlier, CP attenuates the apoptotic events in endothelial cells, which provide further evidence on the vasoprotective potentials for this protein $[73,85]$. These beneficial effects strongly suggest that CP therapy can provide a new therapeutic target against DM-induced vasculopathy as well as for DN [69].

There is similar evidence in renal tissues [86, 87]. Kamikawa and colleagues in 2008 demonstrated similar beneficial effects of CP in renal tissues [86]. They found that with one week of CP therapy in animals with T1DM significantly reversed the DM-dependent NO decline and improved the vascular function by inducing the eNOS activity in renal tissues and suggested that these effects may provide new renoprotective potentials [86]. This effect was 
accompanied by higher eNOS expression in glomeruli and endothelial cells of renal arterioles of diabetic animals [86]. Panero et al. in 2009 conducted a large clinical study on patients with T1DM and found that fasting plasma CP level has a negative correlation with the incidence of vascular complications [59]. Elbassuoni et al., in recent research in 2018, demonstrated that CP prevents DN and improves renal function by NO-dependent mechanism [87]. They reported that $\mathrm{CP}$ replacement therapy might be a novel renoprotective therapy which works by NO synthesis induction and providing vasoprotective effects [87]. Although more studies are still needed, this evidence suggests that the CP can provide vasoprotective effects in the renal tissues [79, 81-83, 87].

\section{Anti-inflammatory effects}

Inflammatory responses have an essential role in the pathophysiology of DN [88, 89]. It has been shown that various pro-inflammatory mediators such as IL (interleukin)-1 $\beta$, IL-6, IL-18, monocyte chemoattractant protein-1 (MCP-1), TNF- $\alpha$, intercellular adhesion molecule 1 (ICAM-1), vascular cell adhesion protein 1 (VCAM-1), E-selectin, different adiponectin, leptin, endothelial cell-selective adhesion molecule (ESAM) and matrix metalloproteinase-2 (MMP-2) are closely associated to the onset and progression of DN [90-92]. Also, studies have suggested that different renal cells as glomerular, endothelial, tubular and mesangial cells express these pro-inflammatory cytokines and release them, which can result in structural and functional injuries in the kidneys $[93,94]$. This evidence has led to the development of the inflammatory hypothesis, which emphasizes the significant role of inflammation in the pathophysiology of DN [90, 95].

Emerging evidence indicates that CP has potent anti-inflammatory properties [96]. It can reduce the recruitment of leukocytes by down-regulation of different adhesion molecules such as p-selectin, ICAM-1 and VCAM-1, which are typically increased during the inflammatory 
process [66, 96, 97]. Consequently, the expression and release of various inflammatory mediators are suppressed, leading to a lower local and systemic inflammation [96]. This effect may be via NF- $\mathrm{B}$ inhibition in both endothelial cells and monocytes [98]. Haidet et al. in 2012 found that CP suppresses the monocytes-dependent inflammatory responses by the inhibition of NF- $\mathrm{BB}$ and down-regulation of various inflammatory cytokines in T1DM [98]. CP is also capable of preventing the DM-induced vascular smooth muscle cell migration, which commonly occurs in diabetic vasculopathy $[64,99]$. Moreover, some studies found a negative correlation between CP plasma concentration and the levels of circulating cytokines in patients with T1DM [100]. This pathway may provide new therapeutic targets against diabetes-induced vascular complications such as DN [58, 101, 102]. Chima and coworkers in 2011 have observed that $\mathrm{CP}$ improves renal function by attenuating the inflammatory responses in hemorrhagic shock [103]. They found that CP potentially down-regulates the c-Fos subunit, ERK 1/2 and JNK (c-Jun N-terminal kinase), leading to a lower expression of cytokines in the kidneys [103].

However, some adverse reports are implying that CP exerts some inflammatory effects [54, 104]. This evidence suggested that $\mathrm{CP}$ has proinflammatory roles in some conditions and thereby, induces inflammatory responses [54, 104]. For example, a recent study has reported that CP is correlated positively with the incidence of inflammatory nonalcoholic fatty liver disease (NAFLD) [105]. So, it seems that more experimental and clinical evaluations are still required in this regard.

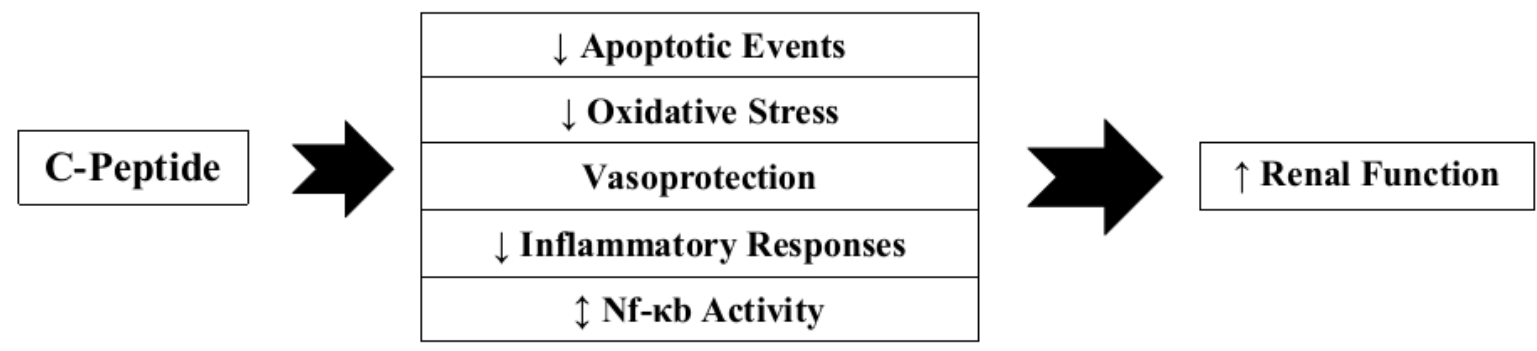


Figure 1: Possible links between C-peptide and renal function in the diabetic milieu

\section{Antioxidant Effects}

Oxidative stress is a major underlying cause of many complications resulting from DM [106, 107]. This deleterious event occurs when the physiologic balance between free radicals and the antioxidant defense system deteriorates toward the production of more free radicals [106]. It has been shown that oxidative stress has a crucial role in various aspects of renal failure by inducing the pathophysiologic events leading to histological injuries [32]. The agents that can potentiate the antioxidative system have always tried for the prevention and treatment of various oxidative stress-dependent states, including DN [38].

There is some evidence demonstrating the antioxidative properties of CP in different tissues [72, 84]. It has been demonstrated that CP potentiates the antioxidative system and protects various tissues against oxidative damages in a diabetic milieu [72, 84]. Ragy et al. in 2019 have shown that CP improved oxidative stress in the pancreatic tissues in TIDM [108]. Also, Cifarelli et al. in 2011 found that CP ameliorates the oxidative stress via lowering the Nox enzyme activity in the endothelial cells [72]. Moreover, Bhatt and coworkers in 2013 reported that $\mathrm{CP}$ reduces the free radical generation and provides antioxidative potentials by an AMPK $\alpha$ dependent mechanism [84].

Furthermore, Luppi et al. in 2014 demonstrated the antioxidant potency of CP in beta cells suggesting that CP can attenuate the oxidative stress in islets [109]. This evidence demonstrates the antioxidative capabilities of CP. Elbassuoni et al. in 2018 provided related evidence in the kidneys, indicating four weeks of CP therapy improved the renal function partially via lowering the oxidative damages in rats with T1DM [87]. 


\begin{tabular}{|c|c|c|c|c|}
\hline \multirow{2}{*}{$\begin{array}{l}\text { Molecular } \\
\text { Mechanisms }\end{array}$} & \multirow{2}{*}{$\begin{array}{c}\text { Renoprotective } \\
\text { Effects }\end{array}$} & \multicolumn{3}{|c|}{ References } \\
\hline & & In vitro & In vivo & Clinical \\
\hline NF-kb modulation & $\begin{array}{l}\text { Modulates Nf-kb } \\
\text { activity and thereby } \\
\text { factors involved in DN } \\
\text { such as TGF- } \beta, \text { Bcl } 2 \text {, } \\
\text { ICAM-1 and TNF- } \alpha\end{array}$ & [64-66] & {$[24,55]$} & - \\
\hline Anti-apoptotic effects & $\begin{array}{c}\text { Reduces apoptotic } \\
\text { events leading to lower } \\
\text { histological injuries in } \\
\text { renal tissues }\end{array}$ & {$[65,72,73]$} & {$[70,71]$} & - \\
\hline Vasoprotective effects & $\begin{array}{c}\text { Provides potent } \\
\text { vasoprotective effects } \\
\text { in renal vasculature } \\
\text { network leading to } \\
\text { improved kidney } \\
\text { function }\end{array}$ & - & {$[86,87]$} & {$[59,78]$} \\
\hline $\begin{array}{c}\text { Anti-inflammatory } \\
\text { properties }\end{array}$ & $\begin{array}{l}\text { Attenuates leucocyte } \\
\text { recruitment and } \\
\text { inflammatory mediators } \\
\text { as P-selectin, ICAM-1, } \\
\text { interleukins and } \\
\text { VCAM- } \\
\text { 1expression/release } \\
\text { leading to lower } \\
\text { inflammation-induced } \\
\text { renal failure }\end{array}$ & {$[98]$} & [103] & $\begin{array}{r}{[100,} \\
103]\end{array}$ \\
\hline $\begin{array}{c}\text { Antioxidative } \\
\text { potentials }\end{array}$ & $\begin{array}{l}\text { May protects renal } \\
\text { tissues against } \\
\text { oxidative damages in } \\
\text { the diabetic milieu }\end{array}$ & {$[72,109]$} & [87] & - \\
\hline
\end{tabular}

Table 2; possible molecular interactions between c-peptide and diabetic nephropathy ( $\mathrm{DN}=$ diabetic nephropathy, TGF- $\beta=$ transforming growth factor- $\beta$, ICAM-1 = intercellular adhesion molecule-1, VCAM-1= vascular cell adhesion molecule- 1 , TNF- $\alpha=$ tumor necrosis factor $-\alpha$ )

\section{Conclusion}

C-peptide is continuously released by the pancreatic $\beta$-cells along with insulin into the circulation and thereby is deficient in states with dysfunctional $\beta$-cells such as T1DM. It has now been accepted that the $\mathrm{CP}$ is not just an inert peptide with structural potency in the proinsulin molecule. CP is also a bioactive peptide with potent physiological functions with 
modulatory effects on various intracellular signaling pathways and thereby provides a new therapeutic target for some of the diabetic complications, including DN. There is growing evidence that c-peptide has a protective potential through anti-oxidative, vasoprotective, antiapoptotic, anti-inflammatory and NF-kb modulatory effects, which enables it as a new target in the prevention and treatment of diabetic vasculopathy as well as diabetic nephropathy. However, more clinical studies are needed to evaluate its therapeutic potential in DN.

\section{Acknowledgment}

The authors are thankful to the "Research center of physiology, Semnan University of medical sciences (Semnan, Iran)" for providing technical supports.

\section{Conflict of Interests}

The authors declare that they have no conflict of interest in this study. 


\section{References}

1. Jansson, S.P., et al., Prevalence and incidence of diabetes mellitus: a nationwide populationbased pharmaco-epidemiological study in Sweden. Diabetic medicine, 2015. 32(10): p. 13191328.

2. Sharma, M., I. Nazareth, and I. Petersen, Trends in incidence, prevalence and prescribing in type 2 diabetes mellitus between 2000 and 2013 in primary care: a retrospective cohort study. BMJ open, 2016. 6(1): p. e010210.

3. Mayer-Davis, E.J., et al., Incidence trends of type 1 and type 2 diabetes among youths, 20022012. New England Journal of Medicine, 2017. 376(15): p. 1419-1429.

4. Forbes, J.M. and M.E. Cooper, Mechanisms of diabetic complications. Physiological reviews, 2013. 93(1): p. 137-188.

5. Fowler, M.J., Microvascular and macrovascular complications of diabetes. Clinical diabetes, 2008. 26(2): p. 77-82.

6. Zimmet, P., et al., Diabetes mellitus statistics on prevalence and mortality: facts and fallacies. Nature Reviews Endocrinology, 2016. 12(10): p. 616.

7. Yaribeygi, H., S.L. Atkin, and A. Sahebkar, Potential roles of microRNAs in redox state: An update. J Cell Biochem, 2018.

8. Kakio, Y., et al., Diabetic nephropathy is associated with frailty in patients with chronic hemodialysis. Geriatrics \& gerontology international, 2018. 18(12): p. 1597-1602.

9. Gallagher, H. and R. Suckling, Diabetic nephropathy: where are we on the journey from pathophysiology to treatment? Diabetes, Obesity and Metabolism, 2016. 18(7): p. 641-647.

10. Wahren, J. and C. Larsson, C-peptide: new findings and therapeutic possibilities. Diabetes research and clinical practice, 2015. 107(3): p. 309-319.

11. Zhu, S., et al., Monitoring C-peptide storage and secretion in islet B-cells in vitro and in vivo. Diabetes, 2016. 65(3): p. 699-709.

12. Leighton, E., C.A. Sainsbury, and G.C. Jones, A practical review of C-peptide testing in diabetes. Diabetes therapy, 2017. 8(3): p. 475-487.

13. Williams, K.V., et al., Persistent C-peptide levels and microvascular complications in childhood onset type 1 diabetes of long duration. Journal of Diabetes and its Complications, 2019.

14. Brunskill, N., C-peptide and diabetic kidney disease. Journal of internal medicine, 2017. 281(1): p. 41-51.

15. Recio, C., et al., Suppressor of cytokine signaling-1 peptidomimetic limits progression of diabetic nephropathy. Journal of the American Society of Nephrology, 2017. 28(2): p. 575-585.

16. Association, A.D., Diagnosis and classification of diabetes mellitus. Diabetes care, 2014. 37(Supplement 1): p. S81-S90.

17. de Faria Maraschin, J., Classification of diabetes, in Diabetes. 2013, Springer. p. 12-19.

18. VanBuecken, D.E. and C.J. Greenbaum, Residual C-peptide in type 1 diabetes: what do we really know? Pediatric diabetes, 2014. 15(2): p. 84-90.

19. Jones, A. and A. Hattersley, The clinical utility of C-peptide measurement in the care of patients with diabetes. Diabetic Medicine, 2013. 30(7): p. 803-817.

20. Hills, C.E. and N.J. Brunskill, Cellular and physiological effects of C-peptide. Clinical science, 2009. 116(7): p. 565-574.

21. Venugopal, S.K. and I. Jialal, C Peptide, in StatPearls [Internet]. 2018, StatPearls Publishing.

22. Yosten, G.L. and G.R. Kolar, The physiology of proinsulin C-peptide: unanswered questions and a proposed model. Physiology, 2015. 30(4): p. 327-332.

23. Rigler, R., et al., Specific binding of proinsulin C-peptide to human cell membranes. Proceedings of the National Academy of Sciences, 1999. 96(23): p. 13318-13323.

24. $\mathrm{Li}, \mathrm{Y}$., et al., C-peptide prevents NF-KB from recruiting $\mathrm{p} 300$ and binding to the inos promoter in diabetic nephropathy. The FASEB Journal, 2018. 32(4): p. 2269-2279. 
25. Galuska, D., et al., C-peptide increases Na, K-ATPase expression via PKC-and MAP kinasedependent activation of transcription factor ZEB in human renal tubular cells. PLoS One, 2011. 6(12): p. e28294.

26. Wahren, J., et al., Role of C-peptide in human physiology. American Journal of PhysiologyEndocrinology And Metabolism, 2000. 278(5): p. E759-E768.

27. Alves, M.T., et al., The dual effect of C-peptide on cellular activation and atherosclerosis: Protective or not? Diabetes/metabolism research and reviews, 2019. 35(1): p. e3071.

28. Wang, L., et al., C-peptide is independently associated with an increased risk of coronary artery disease in T2DM subjects: a cross-sectional study. PLoS One, 2015. 10(6): p. e0127112.

29. Seidell, J.C., et al., Visceral fat accumulation in men is positively associated with insulin, glucose, and C-peptide levels, but negatively with testosterone levels. Metabolism, 1990. 39(9): p. 897-901.

30. Li, Y., et al., Serum C-peptide as a key contributor to lipid-related residual cardiovascular risk in the elderly. Archives of gerontology and geriatrics, 2017. 73: p. 263-268.

31. Pickens, C.A., et al., Obesity, adipokines, and C-peptide are associated with distinct plasma phospholipid profiles in adult males, an untargeted lipidomic approach. Scientific reports, 2017. 7(1): p. 6335.

32. Yaribeygi, H., et al., Oxidative stress induces renal failure: a review of possible molecular pathways. Journal of cellular biochemistry, 2018. 119(4): p. 2990-2998.

33. Bhattacharjee, N., et al., Mechanistic insight of diabetic nephropathy and its pharmacotherapeutic targets: an update. European journal of pharmacology, 2016. 791: p. 824.

34. Soldatos, G. and M. Cooper, Diabetic nephropathy: important pathophysiologic mechanisms. Diabetes research and clinical practice, 2008. 82: p. S75-S79.

35. Umanath, K. and J.B. Lewis, Update on diabetic nephropathy: core curriculum 2018. American Journal of Kidney Diseases, 2018. 71(6): p. 884-895.

36. Yaribeygi, H., S.L. Atkin, and A. Sahebkar, Interleukin-18 and diabetic nephropathy: A review. Journal of cellular physiology, 2019. 234(5): p. 5674-5682.

37. Yaribeygi, H., et al., Fenofibrate improves renal function by amelioration of NOX-4, IL-18, and p53 expression in an experimental model of diabetic nephropathy. Journal of cellular biochemistry, 2018. 119(9): p. 7458-7469.

38. Yaribeygi, H., et al., Crocin improves renal function by declining Nox-4, IL-18, and $p 53$ expression levels in an experimental model of diabetic nephropathy. Journal of cellular biochemistry, 2018. 119(7): p. 6080-6093.

39. Arora, M.K. and U.K. Singh, Molecular mechanisms in the pathogenesis of diabetic nephropathy: an update. Vascular pharmacology, 2013. 58(4): p. 259-271.

40. Chang, A.S., et al., Transforming growth factor- 81 and diabetic nephropathy. American Journal of Physiology-Renal Physiology, 2015. 310(8): p. F689-F696.

41. Ahmed, S., et al., Diosmin modulates the NF-kB signal transduction pathways and downregulation of various oxidative stress markers in alloxan-induced diabetic nephropathy. Inflammation, 2016. 39(5): p. 1783-1797.

42. Reidy, K., et al., Molecular mechanisms of diabetic kidney disease. The Journal of clinical investigation, 2014. 124(6): p. 2333-2340.

43. Yaribeygi, H., et al., Effects of novel antidiabetes agents on apoptotic processes in diabetes and malignancy: Implications for lowering tissue damage. Life sciences, 2019.

44. Molitch, M.E., et al., Nephropathy in diabetes. Diabetes care, 2004. 27: p. S79.

45. Al-Rasheed, N., et al., Potent activation of multiple signalling pathways by C-peptide in opossum kidney proximal tubular cells. Diabetologia, 2004. 47(6): p. 987-997.

46. Zhong, Z., et al., C-peptide stimulates ERK1/2 and JNK MAP kinases via activation of protein kinase $C$ in human renal tubular cells. Diabetologia, 2005. 48(1): p. 187-197. 
47. Giebink, A.W., et al., C-peptide-stimulated nitric oxide production in a cultured pulmonary artery endothelium is erythrocyte mediated and requires $\mathrm{Zn2+}$. Diabetes/metabolism research and reviews, 2013. 29(1): p. 44-52.

48. Hills, C.E. and N.J. Brunskill, C-peptide and its intracellular signaling. The review of diabetic studies: RDS, 2009. 6(3): p. 138.

49. Cifarelli, V., M. Trucco, and P. Luppi, Anti-inflammatory effects of C-peptide prevent endothelial dysfunction in type 1 diabetes. Immunology, Endocrine \& Metabolic Agents in Medicinal Chemistry (Formerly Current Medicinal Chemistry-Immunology, Endocrine and Metabolic Agents), 2011. 11(1): p. 59-70.

50. Al-Rasheed, N.M., et al., Ligand-independent Activation of Peroxisome Proliferator-activated Receptor- $\gamma$ by Insulin and C-peptide in Kidney Proximal Tubular Cells DEPENDENT ON PHOSPHATIDYLINOSITOL 3-KINASE ACTIVITY. Journal of Biological Chemistry, 2004. 279(48): p. 49747-49754.

51. Huang, D.-Y., et al., Human C-peptide acutely lowers glomerular hyperfiltration and proteinuria in diabetic rats: a dose-response study. Naunyn-Schmiedeberg's archives of pharmacology, 2002. 365(1): p. 67-73.

52. Samnegård, B., et al., C-peptide and captopril are equally effective in lowering glomerular hyperfiltration in diabetic rats. Nephrology Dialysis Transplantation, 2004. 19(6): p. 13851391.

53. Stridh, S., et al., C-peptide normalizes glomerular filtration rate in hyperfiltrating conscious diabetic rats, in Oxygen transport to tissue xxx. 2009, Springer. p. 219-225.

54. Vasic, D. and D. Walcher, Proinflammatory effects of C-Peptide in different tissues. International journal of inflammation, 2012. 2012.

55. Samnegård, B., et al., C-peptide prevents glomerular hypertrophy and mesangial matrix expansion in diabetic rats. Nephrology Dialysis Transplantation, 2005. 20(3): p. 532-538.

56. Hills, C.E., N.J. Brunskill, and P.E. Squires, C-peptide as a therapeutic tool in diabetic nephropathy. American journal of nephrology, 2010. 31(5): p. 389-397.

57. Shaw, J.A., et al., C-peptide as a therapy for kidney disease: A systematic review and metaanalysis. PloS one, 2015. 10(5): p. e0127439.

58. Cifarelli, V., Anti-inflammatory properties of C-peptide. A new therapeutic strategy for reducing vascular damage in type 1 diabetes patients. 2011, University of Pittsburgh.

59. Panero, F., et al., Fasting plasma C-peptide and micro-and macrovascular complications in a large clinic-based cohort of type 1 diabetic patients. Diabetes care, 2009. 32(2): p. 301-305.

60. Suryavanshi, S.V. and Y.A. Kulkarni, NF-KB: a potential target in the management of vascular complications of diabetes. Frontiers in pharmacology, 2017. 8: p. 798.

61. Mezzano, S., et al., NF-KB activation and overexpression of regulated genes in human diabetic nephropathy. Nephrology Dialysis Transplantation, 2004. 19(10): p. 2505-2512.

62. Yerra, V.G., et al., Potential therapeutic effects of the simultaneous targeting of the Nrf2 and NF-KB pathways in diabetic neuropathy. Redox biology, 2013. 1(1): p. 394-397.

63. Sanchez, A.P. and K. Sharma, Transcription factors in the pathogenesis of diabetic nephropathy. Expert reviews in molecular medicine, 2009. 11.

64. Cifarelli, V., et al., Human proinsulin C-peptide reduces high glucose-induced proliferation and NF-KB activation in vascular smooth muscle cells. Atherosclerosis, 2008. 201(2): p. 248-257.

65. Al-Rasheed, N.M., G.B. Willars, and N.J. Brunskill, C-Peptide Signals via Gai to Protect against TNF- $\alpha$-Mediated Apoptosis of Opossum Kidney Proximal Tubular Cells. Journal of the American Society of Nephrology, 2006. 17(4): p. 986-995.

66. Luppi, P., et al., Human C-peptide antagonises high glucose-induced endothelial dysfunction through the nuclear factor-kB pathway. Diabetologia, 2008. 51(8): p. 1534.

67. Wahren, J., A. Kallas, and A.A. Sima, The clinical potential of C-peptide replacement in type 1 diabetes. Diabetes, 2012. 61(4): p. 761-772. 
68. Fioretto, P. and M. Mauer. Histopathology of diabetic nephropathy. in Seminars in nephrology. 2007. Elsevier.

69. Bhatt, M.P., Y.-C. Lim, and K.-S. Ha, C-peptide replacement therapy as an emerging strategy for preventing diabetic vasculopathy. Cardiovascular Research, 2014. 104(2): p. 234-244.

70. Li, Z.-g., W. Zhang, and A.A. Sima, C-peptide prevents hippocampal apoptosis in type 1 diabetes. Experimental Diabetes Research, 2002. 3(4): p. 241-245.

71. Sima, A.A. and Z.-g. Li, The effect of C-peptide on cognitive dysfunction and hippocampal apoptosis in type 1 diabetic rats. Diabetes, 2005. 54(5): p. 1497-1505.

72. Cifarelli, V., et al., C-peptide reduces high-glucose-induced apoptosis of endothelial cells and decreases NAD $(P) \mathrm{H}$-oxidase reactive oxygen species generation in human aortic endothelial cells. Diabetologia, 2011. 54(10): p. 2702.

73. Bhatt, M.P., et al., C-peptide prevents hyperglycemia-induced endothelial apoptosis through inhibition of reactive oxygen species-mediated transglutaminase 2 activation. Diabetes, 2013. 62(1): p. 243-253.

74. Kartha, C.C., S. Ramachandran, and R.M. Pillai, Mechanisms of Vascular Defects in Diabetes Mellitus. 2017: Springer.

75. Vallurupalli, S. and J.L. Mehta, Vascular remodeling in diabetes mellitus, in Mechanisms of Vascular Defects in Diabetes Mellitus. 2017, Springer. p. 73-93.

76. Cai, W., et al., MicroRNA-24 attenuates vascular remodeling in diabetic rats through PI3K/Akt signaling pathway. Nutrition, Metabolism and Cardiovascular Diseases, 2019. 29(6): p. 621632.

77. Johansson, B.-L., S. Sjöberg, and J. Wahren, The influence of human C-peptide on renal function and glucose utilization in type 1 (insulin-dependent) diabetic patients. Diabetologia, 1992. 35(2): p. 121-128.

78. Hansen, A., et al., C-peptide exerts beneficial effects on myocardial blood flow and function in patients with type 1 diabetes. Diabetes, 2002. 51(10): p. 3077-3082.

79. Wallerath, T., et al., Stimulation of endothelial nitric oxide synthase by proinsulin C-peptide. Nitric Oxide, 2003. 9(2): p. 95-102.

80. Kitamura, T., et al., Proinsulin C-peptide increases nitric oxide production by enhancing mitogen-activated protein-kinase-dependent transcription of endothelial nitric oxide synthase in aortic endothelial cells of Wistar rats. Diabetologia, 2003. 46(12): p. 1698-1705.

81. Forst, T., et al., Effects of proinsulin C-peptide on nitric oxide, microvascular blood flow and erythrocyte $\mathrm{Na+}, \mathrm{K}+-$ ATPase activity in diabetes mellitus type I. Clinical Science, 2000. 98(3): p. 283-290.

82. Meyer, J.A., et al., Metal-activated C-peptide facilitates glucose clearance and the release of a nitric oxide stimulus via the GLUT1 transporter. Diabetologia, 2008. 51(1): p. 175-182.

83. Johansson, B.-L., J. Wahren, and J. Pernow, C-peptide increases forearm blood flow in patients with type 1 diabetes via a nitric oxide-dependent mechanism. American Journal of PhysiologyEndocrinology and Metabolism, 2003. 285(4): p. E864-E870.

84. Bhatt, M.P., et al., C-peptide activates AMPK $\alpha$ and prevents ROS-mediated mitochondrial fission and endothelial apoptosis in diabetes. Diabetes, 2013. 62(11): p. 3851-3862.

85. Bhatt, M.P., et al., C-peptide protects against hyperglycemic memory and vascular endothelial cell apoptosis. The Journal of endocrinology, 2016. 231(1): p. 97-108.

86. Kamikawa, A., et al., Proinsulin C-peptide abrogates type-1 diabetes-induced increase of renal endothelial nitric oxide synthase in rats. Diabetes/metabolism research and reviews, 2008. 24(4): p. 331-338.

87. Elbassuoni, E.A., N.M. Aziz, and N.F. El-Tahawy, Protective effect of C-peptide on experimentally induced diabetic nephropathy and the possible link between C-peptide and nitric oxide. Applied Physiology, Nutrition, and Metabolism, 2018. 43(6): p. 617-624.

88. Lin, M., et al., Toll-like receptor 4 promotes tubular inflammation in diabetic nephropathy. Journal of the American Society of Nephrology, 2012. 23(1): p. 86-102. 
89. Tuttle, K.R., Linking metabolism and immunology: diabetic nephropathy is an inflammatory disease. 2005, Am Soc Nephrol.

90. Navarro-González, J.F., et al., Inflammatory molecules and pathways in the pathogenesis of diabetic nephropathy. Nature Reviews Nephrology, 2011. 7(6): p. 327.

91. Wada, J. and H. Makino, Inflammation and the pathogenesis of diabetic nephropathy. Clinical science, 2013. 124(3): p. 139-152.

92. Elmarakby, A.A. and J.C. Sullivan, Relationship between oxidative stress and inflammatory cytokines in diabetic nephropathy. Cardiovascular therapeutics, 2012. 30(1): p. 49-59.

93. Nakamura, T., et al., mRNA expression of growth factors in glomeruli from diabetic rats. Diabetes, 1993. 42(3): p. 450-456.

94. Sugimoto, H., et al., Advanced glycation end products-cytokine-nitric oxide sequence pathway in the development of diabetic nephropathy: aminoguanidine ameliorates the overexpression of tumour necrosis factor- $\alpha$ and inducible nitric oxide synthase in diabetic rat glomeruli. diabetologia, 1999. 42(7): p. 878-886.

95. Navarro-Gonzalez, J.F. and C. Mora-Fernandez, The role of inflammatory cytokines in diabetic nephropathy. Journal of the American Society of Nephrology, 2008. 19(3): p. 433-442.

96. Haidet, J., et al., Anti-inflammatory properties of $C$-peptide. The review of diabetic studies: RDS, 2009. 6(3): p. 168.

97. SCALIA, R., et al., C-peptide inhibits leukocyte-endothelium interaction in the microcirculation during acute endothelial dysfunction. The FASEB Journal, 2000. 14(14): p. 2357-2364.

98. Haidet, J., et al., C-peptide reduces pro-inflammatory cytokine secretion in LPS-stimulated U937 monocytes in condition of hyperglycemia. Inflammation Research, 2012. 61(1): p. 27-35.

99. Kobayashi, Y., et al., Human proinsulin C-peptide prevents proliferation of rat aortic smooth muscle cells cultured in high-glucose conditions. Diabetologia, 2005. 48(11): p. 2396-2401.

100. Espes, D., et al., Increased interleukin-35 levels in patients with type 1 diabetes with remaining C-peptide. Diabetes Care, 2017. 40(8): p. 1090-1095.

101. Chowta, M., et al., Serum C peptide level and renal function in diabetes mellitus. Indian journal of nephrology, 2010. 20(1): p. 25.

102. Shaw, J., et al., The therapeutic potential of C-peptide in kidney disease: a protocol for a systematic review and meta-analysis. Systematic reviews, 2014. 3(1): p. 43.

103. Chima, R.S., et al., C-peptide ameliorates kidney injury following hemorrhagic shock. Shock (Augusta, Ga.), 2011. 35(5): p. 524.

104. Vasic, D. and D. Walcher, C-peptide: a new mediator of atherosclerosis in diabetes. Mediators of inflammation, 2012. 2012.

105. Wang, N., et al., C-peptide is associated with NAFLD inflammatory and fibrotic progression in type 2 diabetes. Diabetes/metabolism research and reviews, 2019: p. e3210.

106. Sies, H., C. Berndt, and D.P. Jones, Oxidative stress. Annual review of biochemistry, 2017. 86 : p. 715-748.

107. Domingueti, C.P., et al., Diabetes mellitus: the linkage between oxidative stress, inflammation, hypercoagulability and vascular complications. Journal of Diabetes and its Complications, 2016. 30(4): p. 738-745.

108. Ragy, M.M. and S.M. Ahmed, Protective effects of either C-peptide or I-arginine on pancreatic b-cell function, proliferation, and oxidative stress in streptozotocin-induced diabetic rats. Journal of cellular physiology, 2019. 234(7): p. 11500-11510.

109. Luppi, P. and P. Drain, Autocrine C-peptide mechanism underlying INS1 beta cell adaptation to oxidative stress. Diabetes/metabolism research and reviews, 2014. 30(7): p. 599-609. 